\title{
La bibliothèque des enfants de Clamart fait le pari du
} numérique

The Children's Library of Clamart Bets on Numeric

\section{La biblioteca de los niños de Clamart apuesta a la era digital}

\section{Céline Meyer}

Volume 57, numéro 2, avril-juin 2011

Les services d'information pour les jeunes : actualités et perspectives

URI : https://id.erudit.org/iderudit/1028874ar

DOI : https://doi.org/10.7202/1028874ar

Aller au sommaire du numéro

Éditeur(s)

Association pour l'avancement des sciences et des techniques de la documentation (ASTED)

ISSN

0315-2340 (imprimé)

2291-8949 (numérique)

Découvrir la revue

Citer cet article

Meyer, C. (2011). La bibliothèque des enfants de Clamart fait le pari du numérique. Documentation et bibliothèques, 57(2), 90-100.

https://doi.org/10.7202/1028874ar
Résumé de l'article

Bibliothèque pour enfants pionnière créée en France dans les années 1960, la Petite Bibliothèque Ronde (ex-Joie par les Livres) s'est engagée depuis les années 1990 et surtout 2000 dans un pari qui paraît de moins en moins fou pour une bibliothèque de proximité : celui du numérique. En 2011, il s'agit de doter la bibliothèque d'un environnement numérique pensé pour et avec les enfants issus de la génération numérique, celle-là même qui nous oblige à nous poser les bonnes questions sur le développement des espaces multimédias et à imaginer ce que sera la bibliothèque jeunesse de demain.
Tous droits réservés (C) Association pour l'avancement des sciences et des techniques de la documentation (ASTED), 2011
Ce document est protégé par la loi sur le droit d'auteur. L'utilisation des services d'Érudit (y compris la reproduction) est assujettie à sa politique d'utilisation que vous pouvez consulter en ligne.

https://apropos.erudit.org/fr/usagers/politique-dutilisation/ 


\title{
La bibliothèque des enfants de Clamart fait le pari du numérique
}

\author{
CÉLINE MEYER \\ Conservateur de bibliothèque \\ Directrice, Petite Bibliothèque Ronde \\ celine.meyer@gmail.com
}

\begin{abstract}
RÉSUMÉ | ABSTRACTS | RESUMEN
Bibliothèque pour enfants pionnière créée en France dans les années 1960, la Petite Bibliothèque Ronde (ex-Joie par les Livres) s'est engagée depuis les années 1990 et surtout 2000 dans un pari qui paraît de moins en moins fou pour une bibliothèque de proximité : celui du numérique. En 2011, il s'agit de doter la bibliothèque d'un environnement numérique pensé pour et avec les enfants issus de la génération numérique, celle-là même qui nous oblige à nous poser les bonnes questions sur le développement des espaces multimédias et à imaginer ce que sera la bibliothèque jeunesse de demain.

\section{The Children's Library of Clamart Bets on Numeric}

Created in the 1960s, the Petite Bibliothèque Ronde formerly known as Joie par les livres) is a unique French children's library. Since the 1990s, and more so since 2000 , the library is committed to the numeric format. In 2011, the challenge for the library is to build a numeric environment that takes into accounts the needs and habits of children born in the numeric age. In doing so, we had to rethink how multimedia spaces are designed and to imagine the future of the children's library.

La biblioteca de los niños de Clamart apuesta a la era digital La Petite Bibliothèque Ronde (ex Joie par les Livres) es una biblioteca pionera para niños, creada en Francia en la década de los sesenta. En los años noventa, y en especial en 2000, se comprometió con una apuesta que parecía cada vez menos inverosimil para una biblioteca local : la era digital. En 2011, se buscó dotar a la biblioteca de un entorno digital pensado por los niños provenientes de la generación digital y para ellos. Esta generación es la misma que nos obliga a hacernos preguntas pertinentes acerca del desarrollo de los espacios multimedia y a imaginar cómo será la biblioteca juvenil del futuro.
\end{abstract}

A Petite Bibliothèque Ronde, anciennement Joie par les Livres, a été créée en 1965 par la mécène Anne Gruner Schlumberger avec une petite équipe autour de Geneviève Patte (Blain, Patte et Thurnauer, 2006). Construite par les architectes de l'atelier de Montrouge en plein cour d'une cité sensible dans la région parisienne, elle a été classée monument historique en 2009. À sa création, l'ambition de la bibliothèque des enfants de Clamart était de faire connaitre et reconnaitre l'importance des bibliothèques pour enfants, alors rares en France. Son rayonnement national et international a été immédiat et durable. La bibliothèque a ainsi inspiré de nombreuses initiatives pour encourager l'accès de l'enfant au livre et à la lecture en France et dans le monde. Elle a jeté les bases du Centre National du Livre pour enfants et de la Revue des livres pour enfants, qui ont depuis rejoint le Département Littérature et Art de la Bibliothèque nationale de France.

En février 2007, au cours d'un colloque national tenu à Sciences-po Paris, le directeur du livre et de la lecture français, Benoît Yvert, a évoqué la première mission de la nouvelle politique du livre du Ministère de la Culture : la lutte contre la fracture sociale en matière de lecture publique. Deux bibliothèques publiques sont expressément appelées à rechercher de nouvelles pistes d'action: la Bibliothèque Publique d'Information (BPI) du Centre Pompidou et la bibliothèque des enfants de Clamart qui reprend, sous la présidence de Geneviève Patte puis d'Olivier Ponsoye, le statut associatif qu'elle avait à l'origine. Il s'agit pour celle-ci, en étroite relation avec les bibliothèques qui le souhaitent en France et à l'étranger, de penser et d'expérimenter la bibliothèque des enfants d'aujourd'hui ; d'évaluer et de faire connaître de nouvelles pratiques en s'appuyant sur les modes de vie actuels des enfants et des familles, notamment dans les zones sensibles; d'animer un réseau de coopération et d'échanges entre les professionnels autour d'expériences concrètes, mesurables, transmissibles.

Pionnière jusque dans les années 1980, la bibliothèque des enfants de Clamart est confrontée à une double crise depuis plusieurs années, à l'instar de l'ensemble des bibliothèques publiques françaises : crise de la culture livresque chez les enfants qui lui préfèrent

1. Située au 14, rue de Champagne, Cité de la Plaine, 92140 Clamart, France. 


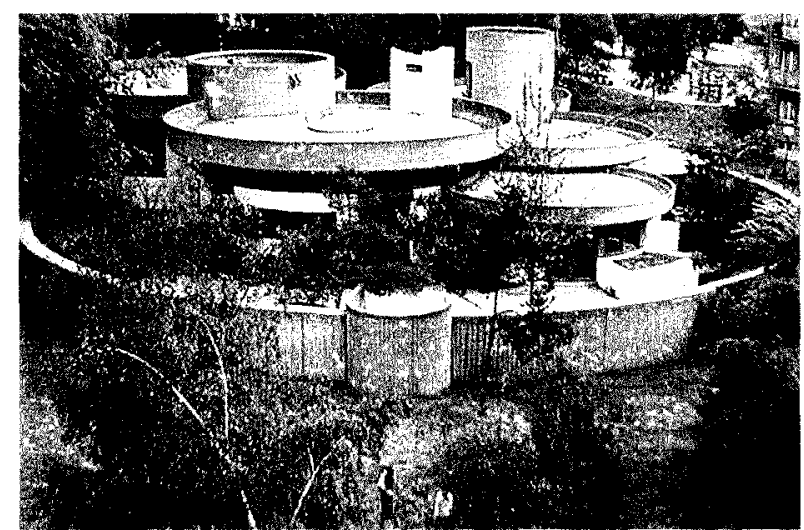

La Petite Bibliothèque Ronde est située au coeur de la Cité de la Plaine à Clamart.

la culture d'écran (Discours de Frédéric Mitterand) et crise de la fréquentation chez les préadolescents et les adolescents qui désertent massivement les bibliothèques, associées à une image studieuse et ringarde (Les 11-18 ans et les bibliotheques municipales). Investie de ses missions pour proposer des stratégies innovantes en matière de lecture publique, la Petite Bibliothèque Ronde s'est engagée dans un pari qui paraît de moins en moins fou pour les petites bibliothèques françaises, celui du numérique, avec pour objectif d'abolir les fractures sociales et culturelles qui lui sont liées et d'accompagner la réflexion des professionnels sur ce sujet qui effraie encore. Pourquoi l'ex-Joie par les Livres a-t-elle choisi de répondre à la crise actuelle par le défi du numérique? Quelles sont ses propositions? Sa démarche pour le gagner?

Première partie, premier combat: convaincre les numérico-sceptiques, ceux pour lesquels seul le livre est légitime des bibliothèques, du bien fondé d'une offre numérique riche dans une bibliothèque pour enfants, en revenant d'abord sur les raisons historiques de l'introduction du multimédia à la bibliothèque de Clamart dans les années 1990 et 2000, puis sur le choix actuel, dans les bibliothèques françaises considérées comme "pionnières ", petites ou grandes, d'un accès numérique adapté aux enfants et contrôlé par les bibliothécaires.

Seconde partie en cours: doter la Petite Bibliothèque Ronde d'un environnement (qui va du site Internet aux jeux vidéo en passant par un catalogue informatique et des livres numériques) pensé pour et avec les enfants issus de la génération numérique, cellelà même qui nous oblige à nous poser les bonnes questions sur le développement des espaces multimédias et à imaginer ce que sera la bibliothèque jeunesse de demain.

\section{Retour sur les raisons d'une offre numérique en bibliothèque}

Les bibliothécaires jeunesse sont comme vous et moi: ils se méfient de ce qu'ils ne connaissent pas et surtout ils croient dans les valeurs de leur métier, en l'occurrence dans le rôle de la littérature jeunesse, dans la capacité des livres à faire grandir les enfants (Patte, 1978). Le livre jeunesse est-il une valeur? Oui, si l'on considère que le livre est un objet parfait en lui-même, comme la roue, la cuillère ou le marteau, dit Umberto Eco (Eco et Carrière, 2009). Il apporte à l'enfant la permanence, la sérénité, l'émotion. La lecture est une expérience que l'on peut qualifier sans exagération d'extraordinaire. Elle est rencontre, partage, éveil au monde (Patte, 1978). Et pourtant... À la bibliothèque des enfants de Clamart, les bibliothécaires n'ont pas attendu le $21^{\mathrm{e}}$ siècle et le "renversement " des valeurs de la culture du livre et de la culture d'écran pour introduire le multimédia.

\section{Premières expériences sur cédéroms dans les années 1990}

La première expérience a démarré très vite, en 1995, avec la consultation de cédéroms sur une poignée d'ordinateurs. Cette expérience pose déjà les principales questions liées à la coexistence du livre et de la machine. La présence d'ordinateurs va-t-elle nuire au calme nécessaire à une salle de lecture? Doit-on leur attribuer une place centrale au risque de porter atteinte à l'image et à l'intérêt du livre? Est-il vraiment justifié de proposer à la bibliothèque des titres comparables à ceux installés sur les ordinateurs et consoles de jeux domestiques?

Si les bibliothécaires de Clamart osent se lancer dans l'aventure du numérique à partir de 1995 - tout en continuant à donner au livre la part du lion - c'est parce qu'ils se sont fixés un triple objectif. D’abord, créer un fonds de cédéroms avec une analyse de contenu pour suivre l'évolution des supports d'information. Puis, permettre aux jeunes du quartier classé zone d'éducation prioritaire de ne pas être défavorisés face à ce nouveau support dans leur vie future. Enfin, étudier les enjeux de l'utilisation des cédéroms et leurs effets sur la lecture des enfants.

Contre toute attente, les enfants vont être très nombreux à s'inscrire (plus de sept cents nouveaux inscrits en trois mois), condition nécessaire pour accéder aux cédéroms installés sur chacun des trois ordinateurs. La nouveauté plaît autant aux filles qu'aux garçons. Les consultations à plusieurs sont fréquentes, entre copains, entre parents et enfants. Elles se déroulent la plupart du temps dans le calme, sans gêner les "lecteurs». Curieusement, l'initiation aux principes de base est très rapide, car là où les adultes veulent tout comprendre, les enfants testent sans hésiter, de manière intuitive, très naturelle, mais également très exigeante, très rigoureuse, comme le décrit une bibliothécaire dans le récit suivant :

"Il est étomnant [...] d'observer la persévérance mise en auvre dans certains cas: Hakim 
et Karim, deux frères plutôt réservés, ont, avec une régularité surprenante, exploré de fond en comble le navire du Mystère du passager clandestin (Nathan Multimédia), jusqu'à en connaître par cour les derniers recoins. C'est à eux que l'on s'adresse désormais pour dépanner les autres. Sabba, elle, passe des après-midi entiers assise derrière les adeptes de L'Odyssée des Zombinis, observant, commentant, conseillant, et se réservant toujours la dernière demi-heure. Ces enfants éprouvent une véritable satisfaction, une certaine fierté même, à maitriser parfaitement un titre. "

(Etchegoyhen, 1997)

Sans surprise, les cédéroms qui obtiennent le plus franc succès sont aussi les plus ludiques. Dans ce domaine, même les titres qui s'adressent aux plus petits séduisent les plus grands. Les bibliothécaires ont vite compris que l'un d'entre eux devait se rendre en permanence disponible pour gérer le planning d'accès aux ordinateurs et pour effectuer les dépannages, fréquents aux débuts de l'informatique dans les bibliothèques françaises. Les professionnels sont également convaincus qu'il n'est pas possible de penser la présence d'ordinateurs dans une bibliothèque sans un atelier multimédia. La fonction de "l'atelier multimédia " est essentielle pour les jeunes publics, car il est l'occasion de créer un événement, de favoriser des rencontres, des échanges, de prendre du recul aussi. Le monde du multimédia est en perpétuelle évolution. Le petit internaute doit pouvoir se tenir au courant des technologies qui changent de plus en plus vite. C'est le rôle de la bibliothèque de lui offrir cette maîtrise du monde (Patte, 1978).

\section{L'arrivée d'internet dans les années 2000}

En 2002, l'environnement va être bouleversé par l'arrivée d'Internet. Pour offrir ce qui prend le nom de "service multimédia ", la bibliothèque de Clamart recrute un "animateur multimédia ». Il est chargé de gérer l'espace avec l'ensemble de l'équipe, chaque membre devant être capable de guider les enfants. Des interrogations apparaissent. D'autres ressurgissent avec une acuité nouvelle. Laccès à Internet doit-il être libre ou contrôlé ? Faut-il intégrer les ordinateurs dans une salle dite multimédia ou les disséminer dans les salles de lecture? Quels usages et quelles animations proposer sur les ordinateurs? Les bibliothèques sont-elles vraiment obligées de s'ouvrir à la floraison de sites et de communautés où l'information, écrite par d'autres internautes, n'est validée par aucune autorité ? L'orientation choisie en 2002 sera d'introduire une offre restreinte, mais de qualité et de faire cohabiter les ordinateurs avec les livres. Le parti pris est de faire du numérique un support naturel de consultation des ressources en bibliothèque, comme le soulignent Isabelle RoseJalaber et Florence Olivier dans un numéro de la Revue des livres pour enfants daté de 2002 :

"Les sites sélectionnés sont considérés comme des ressources supplémentaires qui s'apparentent aux ressources imprimées de la bibliothèque: leur mode de classement est similaire (les sites sont regroupés par sujets rappelant le classement des livres) et ils sont sélectionnés par l'équipe comme le sont les livres (les bibliothécaires chargés d'un secteur d'acquisition pour les livres participent à la sélection de sites dans le même domaine). »

Les bibliothécaires développent une vraie compétence dans la recherche documentaire conçue comme multi-support. Ils sont aptes à accompagner l'enfant dans sa découverte et dans son utilisation du multimédia. Toutefois, les sollicitations des jeunes se limitent essentiellement aux problèmes techniques qu'ils rencontrent (pannes, erreurs...), car ils s'imaginent (à tort!) tout savoir sur Internet et par conséquent n'avoir besoin de personne. Or la toile est un territoire vaste, touffu, semé d'embûches. Le bon usage d'Internet suppose sens du discernement et esprit critique, loin de la simplicité et de la consommation rapide qui collent à l'image du média et qui en constituent un des principaux attraits pour les enfants. C'est la raison pour laquelle le premier devoir du "médiathécaire" est d'orienter les enfants dans leurs recherches vers des ressources qui leur conviennent et de les encourager à s'aventurer au-delà des sites qu'ils connaissent déjà ${ }^{2} \ldots$

Concrètement, ce sont pas moins de huit postes en réseau, répartis dans les trois salles de lecture que compte la bibliothèque, qui fournissent une offre adaptée à l'âge de chaque utilisateur: une offre pour les 2-3 ans dans la "salle des petits", une offre pour les 4-10 ans dans la "salle des moyens» et une offre pour les 9-15 ans et plus dans la "salle des grands". Un ordinateur supplémentaire, relié à une imprimante, est dédié aux recherches libres sur Internet, mais encadrées par un bibliothécaire. L'ensemble de ces services est réservé aux enfants inscrits à la bibliothèque et ayant signé eux-mêmes un règlement : deux fois par semaine, pendant 30 minutes maximum pour faire face à la demande. Ils peuvent consulter sur place une vingtaine de cédéroms et une centaine de sites triés (en fonction de critères tels que la facilité de lecture et de navigation, la qualité graphique et sonore, la complémentarité des médias, la justesse des informations, l'originalité de la démarche de l'auteur, etc.). Les animations sont encore peu diversifiées, mises à part des formations individuelles qui proposent aux enfants, à partir de 9 ans, de mieux comprendre la navigation sur Internet. Les

2. Voir aussi Chaimbault, Thomas. L'offre à distance des bibliothèques pour la jeunesse. 2009. Revue des Livres pour enfants, $\mathrm{n}^{\circ} 247$ (juin) : 130-133. 
parents sont fortement encouragés à se familiariser au multimédia avec leurs enfants. En effet, on pressent que leur méconnaissance de ces outils, investis par les jeunes générations, réduira les possibilités de dialogue au sein des foyers et ouvrira grand la porte aux dangers - bien réels - du monde virtuel3.

\section{Aujourd'hui, le numérique modifie notre modèle de bibliothèque}

Après les cédéroms dans les années 1990, Internet dans les années 2000 , quelle est LA nouvelle technologie qui poussera les bibliothécaires à repenser leur offre, aujourd'hui et dans les années 2010? Est-ce l'opportunité pour les médiathèques de concevoir leur propre site Internet? Faut-il se tourner du côté des jeux vidéo? Des réseaux sociaux? Des blogs? Des sites de partage? S'agit-il plus globalement de toutes les propriétés du Web actuel réunies sous l'appellation générique Web 2.0?

La Petite Bibliothèque Ronde ne prétend pas trancher la question, mais en orientant à un instant $T$ de son histoire - dès 2009 - une grande partie de sa stratégie sur le numérique, en effectuant des arbitrages budgétaires parfois au détriment du livre, en engageant un bibliothécaire chargé des projets numériques, elle va donner des preuves concrètes, visibles, qu'elle parie sur le numérique. Il ne s'agit pas là d'un simple effet de mode, mais bien de l'application d'une intime conviction: les changements induits par le numérique sont tels que c'est notre conception même de la bibliothèque qui est à réévaluer.

Tous les bibliothécaires jeunesse sont actuellement confrontés à la nécessité de ce repositionnement. L'organisation d'une journée d'étude habilement intitulée "Bibliothécaire jeunesse: quel métier?" 4 témoigne de la volonté de prendre en compte ce problème, mais aussi du malaise d'une profession et de l'impossibilité d'y apporter des solutions toutes simples. Le débat n'est pas de savoir s'il existe un métier de «bibliothécaire jeunesse » qui serait différent du métier de « bibliothécaire ", mais plutôt de penser la diversité des publics - et donc du public jeune - pour être en capacité de "rendre des services à des publics" 5 (par opposition aux publics qui nous ressemblent et à la «politique de l'offre » traditionnellement à l'œuvre dans le modèle français).

3. Il faudra attendre 2005 et la création, avec le soutien de la Commission européenne, d'Internet sans crainte, pour que ces dangers soient pris en compte au sein d'un programme national de sensibilisation des jeunes aux bons usages d'Internet: <http://www.internetsanscrainte.fr.>.

4. Organisée par l'École nationale supérieure des sciences de l'information et des bibliothèques (enssib) et la Joie par les livres le 21 octobre 2010 à Villeurbanne.

5. Propos de Tony Di Mascio. « Des jeunes et des bibliothèques? " Demi-journée d'étude organisée par la Bibliothèque publique d'information (Bpi) et la Joie par les Livres le 9 février 2010 à Paris.

\section{Tour d'horizon des bonnes pratiques françaises}

Lors des journées d'étude, il n'est pas toujours aisé de rencontrer les bons interlocuteurs ou de les prendre à part pour discuter longuement avec eux de ce qu'ils font ou de ce qu'ils feraient si on leur en donnait les moyens. Nous avons donc agi comme agissent tous les bibliothécaires lorsqu'ils veulent repenser leur offre en profondeur et enregistrer les bonnes pratiques: ils visitent des bibliothèques. Dans notre cas, ce sont l'animateur multimédia, Nicolas Perisse, et le nouveau bibliothécaire chargé des projets numériques, Jérôme Rivière, qui sont partis en 2010 à la rencontre des responsables multimédias, de leurs pratiques, de leurs idées et surtout de leur vision prospective du numérique pour les enfants (L'offre multimédia en bibliothèque jeunesse).

Sans surprise, leur enquête, associée à celle de Claire Hédin De l'offre numérique pour les enfants dans les bibliothèques, va nous donner la possibilité de nous situer dans la moyenne haute des bibliothèques jeunesse françaises. Résumées en une phrase, ces deux enquêtes disent très simplement ceci : l'offre des médiathèques françaises identifiées comme "pionnières", tout en étant adaptée aux enfants, demeure très timide, très hésitante.

\section{Le choix d'un accès adapté aux enfants et contrôlé par le bibliothécaire}

Dans son étude, Claire Hédin analyse en détail trois exemples d'espaces numériques qui reposent sur des stratégies différenciées: la médiathèque José Cabanis de Toulouse dans le sud-ouest de la France, un «établissement virtuel à part entière », la médiathèque de Viroflay en région parisienne, engagée dans le numérique de proximité et la Bibliothèque nationale de France dont l'offre est avant tout pédagogique. La conservatrice de bibliothèque les compare ensuite à plusieurs modèles étrangers, tels que les Public Libraries anglo-saxonnes, Bibliothèque et Archives nationales du Québec et le projet danois de Children's Interactive Library. Elle souligne les pratiques innovantes de part et d'autre tout en faisant le constat, ni positif ni négatif, que les bibliothèques françaises ont opté pour un accès adapté aux enfants et contrôlé par le bibliothécaire.

De notre côté, nous faisons un bilan similaire dans les bibliothèques que nous avons visitées. À Clamart, en 2010 , les ordinateurs ne sont accessibles que sur inscription à la bibliothèque et sur autorisation auprès d'un animateur multimédia. Ici, comme ailleurs, les sessions sont limitées (en moyenne 30 minutes) et contrôlées par divers moyens : le contrôle parental, le moteur de 
recherche BabyGo, la liste de l'Université de Toulouse ${ }^{6}$, ou encore, à la Petite Bibliothèque Ronde, des modules complémentaires du navigateur comme Web of Trust et le blocage de Facebook, du clavardage, des courriels, du téléchargement, etc.

Pourquoi contrôler l'accès à Internet alors que la plupart des enfants utilisent l'ordinateur tous les jours et fréquentent assidûment les sites de téléchargement et les médias sociaux? Selon les responsables multimédias interrogés, le contrôle de l'accès a pour but de créer dès le départ un lien avec les enfants, de les informer des règles et des dangers d'Internet, de les accompagner et de les guider dans leur approche du média. Limiter le temps d'utilisation de l'ordinateur est également une manière d'inciter l'enfant à se tourner vers d'autres occupations et d'éviter l'assimilation, peu avantageuse selon les bibliothécaires, au cybercafé.

La politique des bibliothèques françaises est plutôt d'adapter chacun des postes, plus ou moins neufs, à l'âge de l'utilisateur et parfois à ses usages (soutien scolaire, visionnage de films). Certains établissements interdisent l'accès aux ordinateurs en dessous d'un certain seuil (par exemple 11 ans à la bibliothèque du Chesnay contre quatre ans à la Petite Bibliothèque Ronde). Il faut bien noter qu'en France la médiathèque est souvent le seul lieu d'accès libre à Internet aux mineurs dans une ville. Consciente de cette place importante sur le territoire, la médiathèque propose aussi bien des jeux que de la recherche documentaire ou de l'aide aux devoirs, sans forcément favoriser telle activité au détriment de telle ou telle autre. Mais tous les établissements ne vont pas offrir des ateliers multimédias. Certains, comme la médiathèque d'Issy-les-Moulineaux, considèrent en effet qu'ils n'ont pas pour mission de former à l'utilisation de l'ordinateur et d'Internet, cette mission relevant de la compétence de l'école?.

\section{L'espace multimédia n'est pas un cybercafé}

Sur quoi va alors se concentrer leur offre? Si offrir un accès à Internet n'a rien de révolutionnaire (cet accès peut être libre comme à Lyon ou restreint à une sélection de sites comme à Noisy-le-Sec${ }^{8}$ ), l'originalité des médiathèques se situe dans leur portail d'accès à Internet. Qu'il s'agisse du site Internet de la bibliothèque ou d'une page Web locale, le portail d'accès présente l'immense intérêt de mettre en valeur des sites classés en fonction de l'âge des enfants et de leurs champs de prédilection. Jeux, livres, cinéma, musique,

6. L'Université de Toulouse diffuse une liste noire d'URLs pour permettre un meilleur contrôle de l'utilisation d'Internet. Cette base est très largement utilisée par les écoles. Blacklists UT1, 〈http://cri.univ-tlse1.fr/blacklists/> (consulté sur Internet le 15 octobre 2010).

7. En France, le Brevet informatique et Internet (B2i) est une attestation, délivrée aux élèves des écoles et des collèges, qui reconnait leur capacité à utiliser les outils informatiques et Internet.

8. Une des sitothèques les plus importantes de France: <www.mediatheque-noisylesec.org/enfant/index.htm.

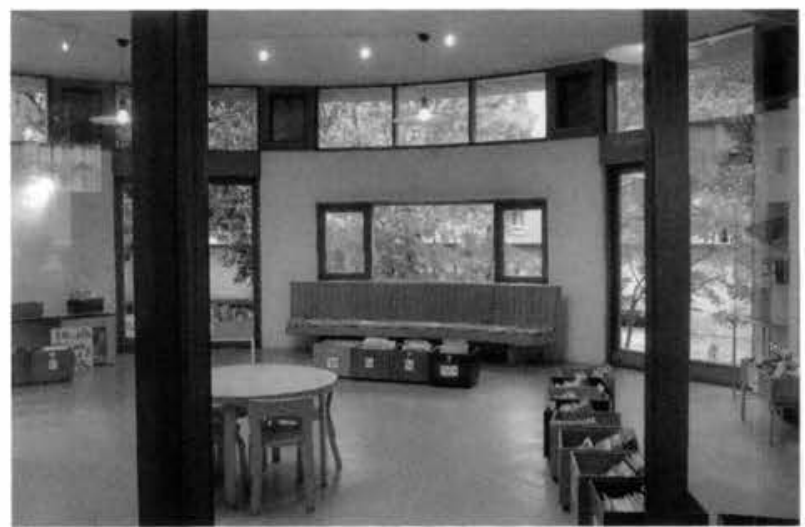

La bibliothèque et une partie du mobilier sont classés depuis 2009.

actualité, vidéos importées de Youtube et de Dailymotion... Mais aussi soutien scolaire, ressources documentaires, apprentissage des langues (notamment Louvre. edu, ToutApprendre, eduMedia, ArteVOD, Jalons...). Parmi les liens recommandés, on retrouve de nombreux sites canadiens, tant pour les jeux que pour les documentaires (Mon JT Quotidien, Sagwa, Cauchemars...).

Les bibliothèques qui proposent des ateliers privilégient les initiations à l'informatique : manipulation de l'ordinateur pour les tout-petits, recherche sur Internet, découverte de quelques logiciels (par exemple l'animation $3 \mathrm{D}$ à Biarritz). Plus rarement, elles conçoivent des ateliers créatifs qui sont intégrés à la programmation générale de l'espace jeunesse ou de l'établissement (animation d'un blog, écriture d'une bande dessinée...). Ces ateliers, à partir de 9-12 ans, peuvent être individuels ou collectifs. Pour l'animateur multimédia de la Petite Bibliothèque Ronde, les deux types d'ateliers doivent coexister. Mais il reconnaît que les ateliers d'initiation à l'informatique, qu'il a un temps proposé le samedi matin, faisaient rarement mouche (à l'exception d'un adulte assidu). Devant cet échec, il a préféré se recentrer, le mercredi après-midi, sur des ateliers de création en lien avec les animations de la bibliothèque : de la Stripsody de Barberian au jeu des sept contes en passant par l'histoire, le conte et même la peinture numériques 9 , certains ateliers sont très suivis et attirent un public fidèle.

Quant aux cédéroms, autrefois proposés en consultation ou au prêt, ils sont souvent abandonnés devant les difficultés d'installation et le désintérêt du public, lié au manque de nouveautés. L’animateur multimédia de la Petite Bibliothèque Ronde en maintient une cinquantaine en consultation pour leur grande qualité (avec un abonnement à la revue Toboclic pour les petits). Enfin, les établissements qui élaborent un blog pour les enfants ${ }^{10}$, des expositions virtuelles, des jeux en réseau

9. Visitez le blog de la Petite Bibliothèque Ronde pour voir les réalisations des ateliers multimédia depuis 2009 : <blog.petitebiblioronde.com. $>$.

10. Medi@zone à Saint-Raphaël (<www.bm-saintraphael.fr/blog/mediazone〉), Graines de critiques à Toulouse (<jeunesse.bibliotheque,toulouse.fr/graines_de_ critiques.html >), le Blog de la Petite Bibliothèque Ronde à Clamart (<blog.petitebiblioronde.com>). 
(à l'instar des tournois Dofus à Saint-Raphaël) ou sur console (comme la Wii à Toulouse, la Wii, la PS 3 et la Xbox 360 à Montpellier) font figure d'exception. Le potentiel ludique des nouveaux médias pour les enfants est encore inégalement exploité dans les bibliothèques françaises.

\section{Comment penser un environnement pour et avec les enfants?}

Qu'en pensent les premiers intéressés? L'offre des médiathèques correspond-elle à leurs attentes? Quelles sont-elles? Les "digital natives", également baptisés "génération $\mathrm{Y}$ » ou « $\mathrm{C}$ », arborent certaines pratiques culturelles communes qui ont été mises en avant par plusieurs enquêtes sur le territoire français. La Petite Bibliothèque Ronde a également mené sa propre étude avant d'élaborer sa stratégie numérique.

\section{Les jeunes et la bibliothèque}

Les enfants nés avec le numérique font un usage massif des technologies de l'information et de la communication. Ils ont un fort niveau d'équipement qui leur permet d'accéder au statut de consommateur de bien culturel au sein du foyer, des habitudes tournées vers la communication et les réseaux sociaux, l'autoproduction des contenus (wiki, blogs), le téléchargement ou le visionnage en streaming (en continu) de musiques et de vidéos... En France, les 8-17 ans passent en moyenne 1,3 heure par jour sur Internet et 9 enfants sur 10 âgés de 6 à 14 ans jouent aux jeux vidéo (Internet sans crainte).

Dématérialisation, culture de la chambre ${ }^{11}$, convergence des écrans ${ }^{12}$ (l'écran de l'ordinateur ou du téléphone devance maintenant celui de la télévision) : selon Sylvie Octobre, les nouvelles technologies «tendent $\dot{a}$ modifier la place attribuée pratiquement et symboliquement aux pratiques culturelles traditionnelles (lecture, fréquentation des équipements culturels) »13. Elles font aussi «évoluer le périmètre du champ culturel, dans le sens d'une plus grande porosité " (développement de l'éclectisme, de la curiosité et de la consommation culturelles). Elles «engendrent dans les jeunes générations une redéfinition de la labellisation au détriment de l'institution et au profit de l'individu et des réseaux». Linéaire et non "zapping », la lecture est aux antipodes des habitudes communicatives et cumulatives (multitâches) des jeunes. Elle souffre d'une érosion généralisée qui a commencé dès la fin de la guerre. Pour essayer de comprendre et d'enrayer ce phénomène, les études sur

11. La culture de chambre désigne l'espace domestique comme étant le pôle central à partir duquel les jeunes vont entrer en relation avec le monde et se construire (Glevarec, 2009)

12. Des jeunes et des bibliothèques? <http://bbf.enssib.fr/consulter/bbf-2010-04 0080-003> (consulté sur Internet le 1o novembre 2010)

13. Pratiques culturelles chez les jeunes et institutions de transmission : un choc des cultures. <http:/www2.culture.gouv.fr/culture/deps/2008/pdf/Cprospectiveog-1. pdf $>$ (consulté sur Internet le 20 mars 2010). les pratiques culturelles des enfants ${ }^{14}$ et sur la lecture se sont multipliées depuis la fin des années 1990 (De Singly, 1989; Poissenot, 1997 ; Octobre, 2004 ; Burgos, 2005 ; Evans, 2009).

La dernière enquête en date, Les 11-18 ans et les bibliothèques municipales (2010), classe les jeunes selon trois profils d'usager : les usagers assidus, les usagers épisodiques et les non-usagers. Les premiers sont dans une relation d'appropriation de la bibliothèque, avec des pratiques traditionnelles telles que l'emprunt, la lecture sur place, les devoirs. Ils ont une vision idéalisée $\mathrm{du}$ lieu sans imaginer pouvoir y trouver une offre plus diversifiée. Non inscrits, les usagers épisodiques sont de «faibles lecteurs " selon l'enquête, mais ils viennent occasionnellement pour "passer le temps » en groupe ou pour réaliser un travail scolaire. Ils ont déjà une image moins classique de la bibliothèque, avec des fonds et des supports plus diversifiés et des horaires élargis en particulier le dimanche. Les non-usagers se satisfont $\mathrm{du}$ CDI (Centre de documentation et d'information en établissement scolaire) et des grandes surfaces commerciales. La raison non avouée de leur non-fréquentation de la bibliothèque est leur manque de goût pour la lecture.

La représentation que tous ces enfants ont de la bibliothèque est assez stéréotypée : un temple du livre, avec ses codes et sa légitimité. L'espace multimédia est cependant leur second espace préféré (30\%), après l'espace $\mathrm{BD}(41 \%)$ et avant l'espace musique $(22 \%)$. Même s'ils ont déjà un ordinateur à la maison, ils aiment utiliser les postes informatiques de la bibliothèque, en particulier dans le cadre de recherches $(34 \%)$, pour jouer (30\%) ou pour d'autres loisirs $(25 \%)$. Ils voudraient néanmoins avoir un accès moins contraint aux ordinateurs, sans réservation préalable, sans limite de temps et avec la possibilité de surfer librement sur tous les sites Internet. Quand ils ont une recherche à faire pour leur travail scolaire, $69 \%$ des 11-14 ans utilisent Internet et quand ils ont du temps libre, utiliser l'ordinateur arrive juste après être avec des copains (respectivement $61 \%$ et $63 \%$ ) et avant lire (60\%), écouter de la musique $(55 \%)$ ou regarder la télévision et des DVD ( $52 \%)$. Seulement $8 \%$ des enfants pensent qu'avec Internet, il n'y a plus du tout besoin de lire de livres.

Pour mieux connaître les pratiques de notre public spécifique, nous avons tenté de mener notre propre enquête ${ }^{15}$, en faisant appel à une "grande école $»^{16}$. Bien que perfectible, l'enquête donne quelques orientations utiles. Ainsi, les jeux vidéo arrivent en tête des activités préférées chez les moins de 12 ans. La musique se situe

14. Pour une bibliographie exhaustive par Cécile Touitou et Aline Eisenegger consulter: <http://editionsdelabibliotheque.bpi.fr/Iivre/?GCOI $=84240100$ $884420 \&$ fa $=$ complements $>$.

15. Enquête sur les pratiques culturelles des enfants du quartier de la cité de la Plaine, 2009. Non publiée.

16. Sous l'appellation "grande école» sont regroupées les écoles françaises d'ingénieurs, de management et de haut enseignement. Elles sont caractérisées par le niveau élevé de leur diplôme et par une forte sélection à l'entrée. 


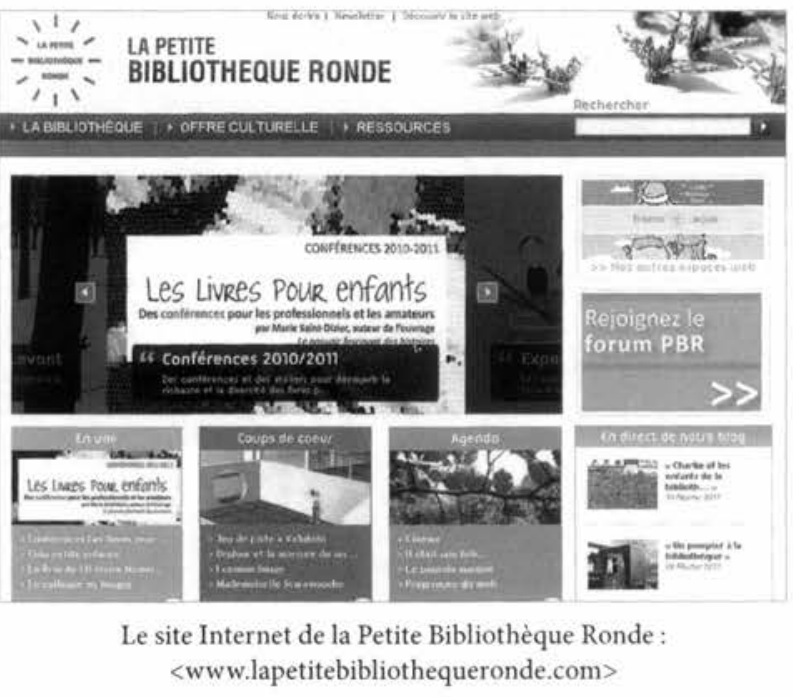

systématiquement dans le top 3 chez les 4-17 ans tandis que la part occupée par la télévision et par l'informatique va augmenter avec l'âge des enfants. La lecture, en seconde position chez les 4-9 ans, n'est plus qu'en huitième position chez les plus grands : $70 \%$ des enfants déclarent aimer lire, mais $50 \%$ évoquent l'obligation par l'école ou lire en situation d'ennui.

Deux tiers des plus de 12 ans utilisent l'ordinateur tous les jours. Ils fréquentent assidûment les moteurs de recherche et les interfaces branchées (YouTube, Myspace, Facebook, etc.), ainsi que les blogs. Si on demande aux enfants de classer leur mode d'utilisation de l'ordinateur, le visionnage de vidéos arrive en tête, puis les jeux vidéo et le courriel. Chez les 12-17 ans, le chat ou clavardage arrive en première position, suivi du téléchargement de musique, les jeux vidéo et les films. À la bibliothèque, les 8-17 ans déclarent retrouver des copains, emprunter des livres, passer le temps, faire des recherches et utiliser Internet. Interrogés sur ce qu'ils aimeraient y faire, ils répondent : regarder des films, écouter de la musique et participer à des ateliers (de création, de photo, de peinture)...

\section{Le colloque sur les pratiques culturelles des enfants et le numérique}

On regrettera qu'aucune des études mentionnées ci-dessus ne revête le caractère systématique de l'enquête sur les pratiques culturelles des Français d'Olivier Donnat (2009) qui porte sur les personnes âgées de 15 ans et plus. De là à comprendre pourquoi, malgré la prolifération des enquêtes, les bibliothécaires se déclarent incapables de savoir ce que les enfants attendent d'un espace multimédia, si les propositions de l'institution parviennent à satisfaire leurs besoins... il n'y a qu'un pas. En l'absence de statistiques fiables, actuelles, exhaustives, beaucoup de bibliothécaires français continuent à limiter leurs informations aux observations sur le terrain. Aussi, les professionnels les plus pessimistes que nous avons rencontrés craignent qu'en l'absence d'une évolution radicale de l'offre, les enfants ne désertent définitivement les espaces multimédias des bibliothèques. De mieux en mieux équipés chez eux, accros aux dernières technologies, de la console au téléphone portable qui sont les grands absents des bibliothèques, les jeunes se lasseront des ordinateurs bridés, lents et vite obsolètes des bibliothèques. La situation est d'autant plus complexe qu'il ne suffira pas de proposer des ordinateurs derniers cris, en accès aussi libre qu'à la maison, pour enrayer la fuite des enfants.

L'enjeu peut se définir en ces termes: si les pratiques culturelles des enfants s'affranchissent des lieux de médiation traditionnels et si tout est consommable à distance, quels services proposer pour continuer à attirer et à conserver le jeune public ? Comment faire prendre un virage aux espaces multimédias quand les bibliothécaires français eux-mêmes se désintéressent massivement du sujet? En effet, peu d'entre eux ont suivi une formation adéquate, ou se considèrent comme compétents face aux enfants nés dans la marmite du numérique. Et ceux qui ont des idées innovantes se sentent isolés au milieu des "pro-livres". À la question "quel est selon vous l'avenir du numérique en médiathèque? ", c'est le blanc... Pour sortir les professionnels de leur mutisme, la Petite Bibliothèque Ronde a mûri l'idée d'un colloque ${ }^{17}$ qui ferait le point sur les réponses des établissements accueillant le jeune public aux nouvelles pratiques culturelles des enfants à l'ère du numérique. Avec en filigrane l'évocation d'un scénario catastrophe possible : la disparition des enfants-lecteurs au $21^{\mathrm{e}}$ siècle !

Organisé le 23 septembre 2010, sous le patronage du ministère de la Culture et avec le soutien de la Caisse des Dépôts (elle gère en France le grand emprunt national sur le développement de l'économie numérique), le colloque et le forum, créés peu après, ont pour première ambition d'offrir un lieu aux professionnels du livre et de l'enfance pour engager une vraie réflexion et échanger leurs expériences. Des bibliothécaires, des éditeurs, des professeurs, des médiateurs, de toute la France et d'ailleurs (Royaume-Uni, Danemark, Colombie, Burkina Faso...), ont fait le déplacement à Paris. S'il s'adresse à tous ceux qui se posent des questions sur l'évolution de la culture (la culture des enfants, c'est un peu celle des adultes de demain), le colloque s'est concentré sur les problématiques qui préoccupent les médiateurs du livre.

\section{Malaise chez les bibliothécaires}

Le premier cri d'alarme a été lancé par Bruno Jammes, directeur adjoint de la Bibliothèque des

\footnotetext{
17. Le colloque est accessible en ligne sur notre site Internet. Les nouvelles pratiques culturelles des enfants face au numérique. $Y$ aura-t-il encore des enfants lecteurs au $21^{c}$ siècle? <http://www.lapetitebibliothequeronde.com/La-bibliotheque/ Activites-Projets/Colloque-Les-nouvelles-pratiques-culturelles-des-enfants-faceau-numerique>(consulté sur Internet le 15 novembre 2010).
} 
sciences et de l'industrie : nous manquons cruellement de données quantitatives et objectives sur les usages, les besoins et les attentes des enfants par rapport au multimédia. Or souligne-t-il « il existe une véritable fracture numérique chez les enfants. Ce n'est pas parce qu'ils sont nés avec les nouvelles technologies que spontanément, de manière innée, ils savent les utiliser ». De telles données intéresseraient donc non seulement les bibliothécaires, mais aussi les éditeurs, les éducateurs, les politiques publiques.

Second cri d'alarme : la grande majorité des bibliothécaires n'est pas à l'aise avec l'idée même d'une offre multimédia en bibliothèque. Des espaces multimédias ont été créés dès les années 1990 sans que les responsables ne se soient posé la question de leur gestion et de la formation du personnel. «En dix ans, déplore Axelle Desaint, formatrice au sein de l'association Territoire ${ }_{21}^{18}$, alors que les pratiques des usagers ont considérablement progressé, du côté des bibliothécaires on bute toujours sur le problème de la LÉGITIMITÉ». Cela peut paraitre incroyable aujourd'hui, mais tant qu'on ne passera pas de la question du "pourquoi ? " à celle du « comment? », les espaces multimédias resteront les parents pauvres des médiathèques.

Le sentiment d'absence de légitimité se lit à plusieurs niveaux : l'institution (notamment les services informatiques qui placent la sécurité des réseaux loin devant les services au public), le personnel (qui ne se sent pas compétent dans une fonction pour laquelle il n'a pas reçu de formation) et les enfants eux-mêmes: ces derniers perçoivent le métier de bibliothécaire comme un métier peu dynamique, ennuyeux, poussiéreux et limité aux livres (Repaire et Toitou, 2010). Les bibliothécaires sont les dernières personnes qui pourraient leur apprendre quelque chose sur le multimédia.

\section{Imaginer la bibliothèque de demain}

Pour Axelle Desaint, il ne faut pas s'étonner si l'offre multimédia proposée par les médiathèques ne fonctionne pas auprès des jeunes, si ces derniers ne respectent pas le personnel préposé aux machines: "Viens me rallumer mon ordinateur! Dépêche-toi de me le réparer!». C'est effectivement le genre d'attitude que nous pouvons constater chez certains adolescents à la Petite Bibliothèque Ronde. Selon la formatrice, dire que l'on doit laisser les enfants faire tout ce qu'ils veulent sur les écrans, de la même manière qu'on les laisse libres de choisir leurs livres, est une grave erreur. Mettre par défaut Google en page d'accueil, c'est tenir aux enfants le discours suivant: "Comportez-vous exactement comme si vous étiez à la maison ou avec vos copains!"

18. Lassociation Territoire 21 milite en faveur de l'éducation aux nouvelles technologies et au développement durable. Elle forme les professionnels de l'enfance, du multimédia et du livre aux nouvelles technologies jeunesse et à l'animation d'un espace public multimédia : 〈http://www.territoires21.org/ $\rangle$.
Or à tout point de vue, accéder au Web par Google est une catastrophe en termes d'éducation ! 19

\section{Être ou ne pas être virtuelle?}

Les bibliothèques qui proposent une autre porte d'entrée sur le Web aux enfants sont si rares en France qu'elles se comptent sur les doigts d'une main. La Petite Bibliothèque Ronde en fait partie depuis 2010. Notre choix s'explique également par la volonté de développer une forte identité virtuelle (importante en termes de communication et de proximité avec les professionnels et avec les jeunes) et de mettre en place une plateforme culturelle fractionnée en autant de pratiques.

Ainsi, ce sont pas moins de quatre espaces qui sont gérés par Clémentine Slembrouck, chargée de communication et responsable du site Internet de la Petite Bibliothèque Ronde : un site institutionnel (<www.lapetitebibliothequeronde.com $>$ ) pour promouvoir la vie de la bibliothèque (histoire, mission, agenda, offre culturelle, projets), un portail jeunesse (<www.petitebiblioronde.com $>$ ) pour lui offrir des ressources numériques de qualité (livres, vidéos, jeux, dossiers thématiques), un blog des enfants (<blog.petitebiblioronde.com>) qui est à la fois leur espace d'expression et la vitrine directe de leurs activités à la bibliothèque, un blog de veille pour les professionnels (<www.enfance-lecture.com>) pour communiquer sur l'actualité de notre métier et sur les tendances qui façonnent le monde de l'enfance et de la lecture.

La Petite Bibliothèque Ronde est également présente sur tous les supports qui parlent aux jeunes : Facebook, Twitter, YouTube, Flickr. Dernier-né, le forum (<forum. lapetitebibliothequeronde.com>) a été pensé pour prolonger les questionnements du colloque et parce que nous avions remarqué qu'un espace de discussion uniquement dédié aux professionnels de la jeunesse faisait défaut. En six mois de fonctionnement, le succès est au rendez-vous puisque la plateforme hors forum totalise 25000 connexions sur tous les continents. Il y a néanmoins de fortes inégalités entre les espaces destinés aux professionnels et ceux pour les enfants très faiblement investis (le portail jeunesse totalise moins de $8 \%$ des connexions). De multiples éléments de réponse viennent à l'esprit pour rendre compte de ces différences, mais les trois principaux ont trait au référencement, à la cible et au contenu des espaces enfants pour lesquels la copie est en partie à revoir !

19. Quelle porte d'entrée pour les enfants sur Internet? <www.territoires21.org/ index.php/google> (consulté sur Internet le 15 mai 2010). Axelle Desaint suggère aussi d'autres outils de recherche pour les moins de 12 ans: <www.kidadoweb.

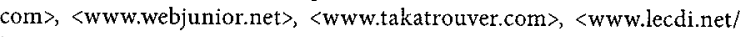
junior $>$. 


\section{Un catalogue innovant pour les enfants}

Ce qui a fait défaut dans la phase de conception de ces espaces jeunesse, c'est l'association des jeunes - principaux intéressés - au projet. Afin de ne pas répéter cette erreur initiale, Fabrice Papy (chercheur en sciences de l'information et de la communication à l'Université de Nancy), Jérôme Dinet (chercheur en psychologie et en ergonomie cognitives à l'Université de Metz) ${ }^{20}$ et Caroline Simon (bibliothécaire chargée de la politique documentaire à la Petite Bibliothèque Ronde) travaillent actuellement sur un outil de recherche documentaire adapté aux jeunes lecteurs en les intégrant et en les associant à toutes les phases de conception, de la rédaction du cahier des charges aux tests utilisateurs des différentes versions. Cette démarche renverse nos habitudes de travail puisque généralement l'usager final n'est considéré qu'à la toute fin du processus de conception des outils et des interfaces. L'un des intérêts de ce projet de catalogue - et non des moindres sera de déterminer les particularités et les spécificités des comportements lorsque ce sont des enfants qui effectuent le processus de recherche documentaire. Il faut bien comprendre que dans un monde régi par les nouvelles technologies, la recherche documentaire est essentielle pour un enfant, puisque l'accès au savoir dépend en grande partie de la réussite lors de cette étape. Les logiciels de recherche documentaires sont le premier pas vers l'information aussi bien sous forme électronique que sous forme papier.

L'utilité d'un catalogue pour les enfants est donc bien réelle : ni un moteur de recherche comme Google ni une solution clef en main proposée par un fournisseur informatique ne pourront le remplacer. Accessible en ligne à partir du portail de la bibliothèque ou sur place à partir d'une console de jeu vidéo, ce catalogue réunira toutes les fonctionnalités aujourd'hui incontournables d'un catalogue de nouvelle génération (Amar et Mesguich, 2009): convivialité-accessibilité (graphisme, nuage de tags pour une navigation hypertexte, recherche simple "à la Google " et recherche avancée par titre, auteur, mais aussi couleur, taille, etc.), participation-proximité (critique, notation, popularité, recommandation), dissémination-visibilité (flux RSS, affichages de première et quatrième de couverture, extraits multimédias, indexation des notices sur le Web par les moteurs de recherche, réutilisation des données par d'autres internautes).

\section{Le prix du livre numérique jeunesse}

Les ressources proposées seront ainsi mixtes, comprenant les livres, revues, cédéroms, de la bibliothèque physique, mais aussi les contenus numériques créés par les internautes et les bibliothécaires et toutes les autres ressources accessibles en ligne (livres numériques, histoires lues multilingues, coups de cœur, images, vidéos, etc.). Ladjectif " autres" est véritablement le plus approprié, car on ne sait pas aujourd'hui à quoi ressemblera le livre numérique pour enfants de demain ${ }^{21}$, ce faisant on utilise un mot du passé pour désigner un objet du futur ${ }^{22}$. On commence à peine à en voir les formidables potentialités avec les e-readers (liseuses) et l'iPad d'Apple (lecture audiovisuelle, interactivité, «transmedia »...). Mais sur le marché des livres jeunesse francophones, en particulier des albums, l'offre des éditeurs est beaucoup trop frileuse: quel sens cela peut-il avoir pour un enfant d'aujourd'hui de tourner les pages virtuelles d'un faux livre?

Persuadée que le livre numérique jeunesse sera un objet hybride ou ne sera pas, la Petite Bibliothèque Ronde a lancé, le 23 septembre 2010 , en partenariat avec Rue des écoles ${ }^{23}$ et les Éditions Circonflexe, le Prix du livre numérique jeunesse. Il s'agit d'un prix littéraire destiné aux écrivains, illustrateurs, graphistes professionnels et amateurs, ainsi qu'aux enfants, qui récompensera la meilleure histoire numérique jeunesse, c'està-dire un texte, une vidéo, une animation (les formes sont libres) racontant une histoire et diffusable sur un support numérique (ordinateurs, téléphones intelligents, etc.).

À notre connaissance, il s'agit du tout premier prix entièrement consacré à la création littéraire numérique jeunesse. Les enfants de Clamart participeront à ce concours en créant leur propre histoire numérique, avec l'aide de Nicolas Perisse, l'animateur multimédia et de la Souris qui Raconte, une toute nouvelle maison d'édition numérique.

\section{Pourquoi nous jouerons aux jeux vidéo à la bibliothèque}

Ce nouvel objet - feu le livre numérique - que les éditeurs, les enfants et les auteurs doivent inventer de concert, ressemble fort, par bien des aspects, à un objet existant et très apprécié des jeunes : le jeu vidéo. Car qu'est-ce qu'un jeu vidéo ? Selon Stéphane Natkin, directeur de l'école nationale du jeu et des médias interactifs numériques et commissaire de l'exposition

\footnotetext{
21. Même s'il existe quelques expérimentations réussies comme les livres jouables et interactifs d'Hervé Tullet : 〈http://tullet.free.fr/>.

22. Colloque : L'avenir du livre pour la jeunesse, organisé par la $\mathrm{BnF}$, la Joie par les livres et l'Afreloce le 26 novembre 2009 à Paris.

23. Rue des écoles est un éditeur indépendant qui met à disposition des élèves et des enseignants des outils novateurs d'enseignement et d'apprentissage.
} 


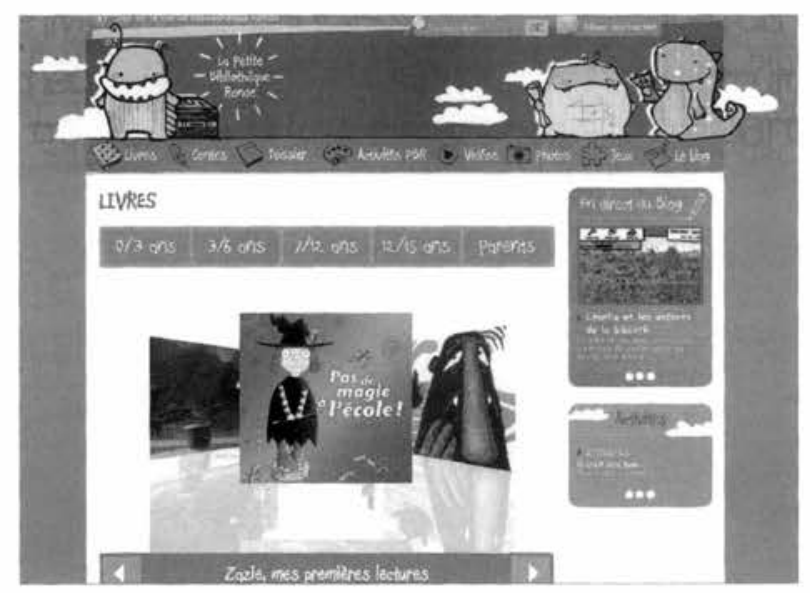

Capture écran 2 : Le portail jeunesse : <www.petitebiblioronde.com>

MuseoGames ${ }^{24}$, c'est une " œuvre interactive dont l'objectif premier est de distraire ses utilisateurs et qui utilise pour sa reproduction un appareil basé sur une technologie informatique ». De fait, le jeu vidéo fait interagir une large série de moyens : la narration, le graphisme, la cinématique, la mise en scène, la musique, mais également le "gameplay" qui traduit l'immersion dans un univers. Contrairement au livre et au cinéma, le jeu vidéo implique totalement; il engendre des décisions réelles sur les actions de l'histoire créant une lecture singulière... On parle de "narration interactive " et d'" engagement vidéo-ludique». Le jeu vidéo est une matière à penser : penser le monde et se penser soi-même ${ }^{25}$. Davantage que la lecture proposée initialement par ses créateurs, ce qui compte c'est bien l'expérience émotionnelle, interactive, vécue par le joueur et qui en fait un média aussi riche sinon plus riche que les précédents.

Le projet de la Petite Bibliothèque Ronde permettra aux enfants et aux familles de jouer ${ }^{26}$ aux meilleurs des jeux vidéo, aussi bien contemporains qu'historiques, et de découvrir de nouvelles expériences de jeu, individuelles et collectives. L'objectif est également de convaincre tant les politiques que les bibliothécaires français de l'intérêt des jeux vidéo en bibliothèque. En effet, alors que les collections de jeux vidéo font partie des bibliothèques américaines et canadiennes depuis un moment, en France il faut encore expliquer que loin d'être réduit, le rôle du bibliothécaire restera essentiel, puisque les jeux vidéo et les supports numériques, comme tous les autres supports, seront choisis pour leurs qualités et intégrés dans une politique documentaire menée par des experts. Pour l'instant les usagers se contentent de jouer à des jeux flash gratuits

24. L'exposition MuseoGames s'est tenue au Musée des arts et métiers à Paris du 22 juin 2010 au 13 mars 2011 : 〈http://museogames.com/>.

25. Êcoutez les interventions de Yann Leroux, psychanalyste, et de Karen Bertrams, consultante pour ProBiblio, durant le colloque sur Les nouvelles pratiques culturelles des enfants face au numérique.

26. A quelques exceptions près, le prêt de jeux vidéo sur console n'est pas encore légal dans les bibliothèques françaises, mais aucune loi ne s'oppose à la consultation de jeux sur place. Des jeux vidéo à la bibliothèque. <http://www.enssib.fr/bibliotheque-numerique/document-2102> (consulté sur Internet le 15 juillet 2009). souvent médiocres. Ce qui changera la donne à la Petite Bibliothèque Ronde, c'est l'achat de consoles de jeu variées (Playstation 3, Wii, Nintendo DSi) et l'acquisition de nombreux titres incontournables ou plus confidentiels, de Max et les Maximonstres à Pro Evolution Soccer. Néanmoins, c'est surtout la proposition d'actions pensées dans une perspective plus globale qui fera la différence auprès du public jeune: ainsi les tournois, les jeux en réseau, les projections sur grand écran, les ateliers multijeux, les événements festifs et les rencontres avec des invités, dans la ville, dans les écoles...

En effet, ce qui est en jeu, c'est bien l'image de la bibliothèque, en particulier auprès des jeunes qui la désertent ou qui ne la fréquentent qu'occasionnellement parce qu'elle semble étrangère, "pas pour eux ». Comme le souligne Céline Ménéghin, auteur Des jeux vidéo à la bibliothèque : "Une politique culturelle bâtie autour du jeu vidéo est loin d'être anecdotique. Elle touche à des enjeux cruciaux en termes de philosophie d'établissement et de publics ". Les animations et ateliers autour d'une console (elle prend l'exemple de la Wii) sont "basés sur l'amusement, la découverte et l'expérience ludique à plusieurs. Cela contribue à faire des bibliothèques des lieux de vie, de convivialité entre les usagers et avec les personnels" (Ménéghin, 2010).

Les jeux vidéo, comme les autres médias numériques, participent de l'image moderne et positive de la nouvelle bibliothèque, capable de satisfaire à la fois les besoins de divertissement et les besoins intellectuels de ses usagers, les enfants pouvant passer, naturellement, comme à la maison - et mieux qu'à la maison - de l'un à l'autre, des loisirs à l'étude, du livre au jeu multimédia.

\section{Conclusion}

Ni Internet ni le livre numérique, les blogs, les jeux vidéo, ne sont des solutions miracle pour attirer les enfants dans notre établissement, mais ces nouvelles technologies légitiment la bibliothèque comme lieu de vie et de convivialité, en phase avec son temps et ouverte aux pratiques culturelles des jeunes. Le temps de la bibliothèque d'étude silencieuse - qui n'a jamais vraiment eu cours à Clamart - est révolu. Celui d'une bibliothèque lieu de vie et de sociabilité où les enfants pourront se regrouper, parler à voix haute, jouer, manger, écouter de la musique, courir, chanter, rire, danser, est à venir.

Dans son intervention au colloque sur L'avenir du livre pour la jeunesse, Thomas Chaimbault affirme que la bibliothèque jeunesse ne sera plus un lieu contraignant 27 : "Elle devient le prolongement de la chambre, du salon, un lieu de rencontre et de retrouvailles avec les copains. L'idée étant de partir des pratiques mêmes des

\footnotetext{
27. Voir également les interventions de Sergio Dogliani, directeur général des Idea Stores et de Jannick Mulvad, concepteur de la Children's Interactive Library au colloque sur Les nouvelles pratiques culturelles des enfants face au numérique.
} 
jeunes pour leur proposer quelque chose qui les intéresse et où ils peuvent se reconnaître. " Ceci en assumant le "côté divertissant de la bibliothèque", en répondant au «besoin informationnel au moyen des nouveaux médias» et en proposant "de nouvelles formes de médiation ". Alors le numérique est-il véritablement un pari qu'une bibliothèque petite ou grosse, francophone ou anglophone, peut choisir de faire ou de ne pas faire? Non! Le numérique est une valeur sûre, aussi incontournable que le livre, dans laquelle la Petite Bibliothèque Ronde a bien l'intention d'investir, d'expérimenter et d'innover, quitte à tâtonner et à se tromper parfois ! 0

\section{Sources consultées}

Amar, Muriel et Véronique Mesguich. 2009. Le Web 2.o en bibliothèques : quels services? quels usages? Paris : Cercle de la librairie.

Blacklists UT1. <http://cri.univ-tlse1.fr/blacklists/> (consulté le 15 octobre 2010).

Blain, Catherine, Geneviève Patte et Gérard Thurnauer. 2006. Espace d lire. La bibliothèque des enfants de Clamart. Paris : Gallimard.

Burgos, Martine. 2005. Le parcours du jeune lecteur dans la bibliothèque : médiation et obstacles. Lecture Jeune (décembre).

Chaimbault, Thomas. 2009. L'offre à distance des bibliothèques pour la jeunesse. Revue des Livres pour enfants, $\mathrm{n}^{\circ} 247$ (juin) : 130-133.

De l'offre numérique pour les enfants dans les bibliothèques. $<$ http://www.enssib.fr/bibliotheque-numerique/notice-48580> (consulté le 15 octobre 2010).

De Singly, François. 1989. Lire à 12 ans. Paris : Nathan.

Des jeux vidéo à la bibliothèque. <http://www.enssib.fr/bibliotheque-numerique/document-2102> (consulté le 15 juillet 2009).

Dinet, Jérôme. 2006. L'ergonomie des sites Web pour et par des jeunes. Revue AC-TICE : 34-36.

Dinet, Jérôme. 2009. Pour une conception centrée-utilisateurs des bibliothèques numériques. Communication \& langages, $\mathrm{n}^{\circ} 161$ : 59-74.

Donnat, Olivier. 2009. Les pratiques culturelles des Français à l'ère du numérique. Paris: La découverte.

Eco, Umberto et Jean-Claude Carrière. 2009. N'espérez pas vous débarrassez des livres! Paris: Grasset.

Etchegoyhen, Mayalen. 1997. L'arrivée des cédéroms à la bibliothèque des enfants de la Joie par les livres. Revue des livres pour enfants, $n^{\circ} 175-176$ (juin) : 52-58.

Evans, Christophe. 2009. Les «Huitdouzan», quelle place en bibliothèque? Revue des Livres pour enfants, $n^{\circ} 248$ (septembre)

Glevarec, Hervé. 2009. La Culture de la chambre. Paris : DEPSMinistère de la Culture.

Internet sans crainte. $<\mathrm{http}$ ://www.internetsanscrainte.fr/> (consulté le 15 octobre 2010).
Ménéghin, Céline. 2010. Des jeux vidéo à la bibliothèque : une nouvelle génération de collections. Bulletin des bibliothèques de France $n^{\circ} 3: 56-60$.

Mitterand, Frédéric. Discours de Frédéric Mitterrand, ministre de la Culture et de la Communication, prononcé à l'occasion de la présentation des 14 propositions pour le développement de la lecture. <http://www.culture.gouv.fr/mcc/Actualites/A-la-une/ Un-plan-pour-developper-la-lecture ?> (consulté le 10 avril 2010).

Octobre, Sylvie. 2004. Les loisirs culturels des 6-14 ans. Paris : La documentation française.

L'offre multimédia en bibliothèque jeunesse. <http://www.enssib. $\mathrm{fr} / \mathrm{breves} / 2011 / 02 / 15 / 1$-offre-multimedia-en-bibliothequejeunesse> (consulté le 17 février 2011).

Patte, Geneviève. 1978. Laissez-les lire! Les enfants et les bibliothèques. Paris: Gallimard

Poissenot, Claude. 1997. Les adolescents et la bibliothèque. Paris : Bibliothèque publique d'information.

Pratiques culturelles chez les jeunes et institutions de transmission : un choc des cultures. <http://www2.culture.gouv.fr/culture/ deps/2008/pdf/Cprospectiveog-1.pdf> (consulté le 20 mars 2010).

Quelle porte d'entrée pour les enfants sur Internet ? <www.territoires21.org/index.php/google> (consulté le 15 mai 2010).

Repaire, Virginie et Cécile Toitou. 2010. Les 11-18 ans et les bibliothèques municipales. Paris: Bibliothèque publique d'information. <http://editionsdelabibliotheque.bpi.fr/livre/ ?GCOI $=842$ $40100884420 \& \mathrm{fa}=$ complements $>$ (consulté le 15 octobre 2010)

Rose-Jalaber, Isabelle et Florence Oliver. 2002. À Clamart, premiers pas sur la toile. Revue des livres pour enfants, $n^{\circ} 208$ (décembre) : 79-83.

\section{Journées d'étude et colloques}

Journée d'étude : Bibliothécaire jeunesse quel métier ? organisée par l'École nationale supérieure des sciences de l'information et des bibliothèques (enssib) et la Joie par les livres le 21 octobre 2010 à Villeurbanne.

Journée d'étude : Des jeunes et des bibliothèques ? organisée par la Bpi et la Joie par les Livres le 9 février 2010 à Paris. <http:// bbf.enssib.fr/consulter/bbf-2010-04-0080-003> (consulté le 10 novembre 2010).

Journée d'étude : Culture numérique : Nouveaux espaces d'expression et de création adolescentes organisée par Lecture jeunesse à la Cité des sciences et de l'Industrie, le 15 septembre 2009.

Colloque : Les nouvelles pratiques culturelles des enfants face au numérique. $Y$ aura-t-il encore des enfants lecteurs au $21^{\mathrm{c}}$ siècle ? <http://www.lapetitebibliothequeronde.com/ La-bibliotheque/Activites-Projets/Colloque-Les-nouvellespratiques-culturelles-des-enfants-face-au-numerique> (consulté le 15 novembre 2010).

Colloque TICE : L'utilisation des moteurs de recherche par les jeunes, organisé à Strasbourg, du 6 au 8 décembre 2010.

Colloque : L'avenir du livre pour la jeunesse, organisé par la $\mathrm{BnF}$, la Joie par les livres et l'A freloce le 26 novembre 2009 à Paris. 